\title{
CONGRUENCE PROPERTIES OF COEFFICIENTS OF MODULAR FORMS FOR $\Gamma_{0}^{+}(5)$
}

\author{
SoYoung ChOI*
}

\begin{abstract}
We find congruence properties on the coefficients of modular forms for $\Gamma_{0}^{+}(5)$ generated by $\Gamma_{0}(5)$ and a Fricke involution $\left(\begin{array}{cc}0 & -1 \\ 5 & 0\end{array}\right)$
\end{abstract}

\section{Introduction}

The study of the arithmetic properties of modular forms with integers is an interesting branch in the theory of modular forms (see [3]). Choie, Kohnen and Ono (see [1]) obtained congruence properties for coefficients of modular forms for $S L_{2}(\mathbb{Z})$. In this paper we discover congruence properties on the coefficients of modular forms for $\Gamma_{0}^{+}(5)$ which is generated $\Gamma_{0}(5)$ and a Fricke involution $\left(\begin{array}{cc}0 & -1 \\ 5 & 0\end{array}\right)$. Let $k$ be an even integer. Let $M_{k}\left(\Gamma_{0}^{+}(5)\right)$ the vector space of modular forms for $\Gamma_{0}^{+}(5)$ and $r:=\operatorname{dim} M_{k}\left(\Gamma_{0}^{+}(5)\right)$. Indeed, we have the following.

(1) $M_{2}\left(\Gamma_{0}^{+}(5)\right)=\{0\}$.

(2) $\operatorname{dim} M_{k}\left(\Gamma_{0}^{+}(5)\right)=(k-2) / 4$ if $k \equiv 2(\bmod 4)$ and $\operatorname{dim} M_{k}\left(\Gamma_{0}^{+}(5)\right)=k / 4+$ 1 otherwise. (See Theorem 2.5.2 in [2]).

As usual, we let $\mathbb{H}$ be the complex upper half plane and $q=e^{2 \pi i z}(z \in \mathbb{H})$ and

$$
E_{k}=1-\frac{2 k}{B_{k}} \sum_{n \geq 0} \sigma_{k-1}(n) q^{n}
$$

Received October 14, 2013; Accepted November 05, 2013.

2010 Mathematics Subject Classification: Primary 11E12, 11F11.

Key words and phrases: modular forms, congruences.

This work was supported by Basic Science Research Program through the National Research Foundation of Korea (NRF) funded by the Ministry of Education, Science and Technology (NRF2012R1A1A3011711). 
be an Eisenstein series of weight $k$, where $\sigma_{k-1}(n)$ is the sum of $(k-1)$ st powers of the positive divisors of $n$ and $B_{k}$ is Bernoulli number. For instance,

$E_{4}(z)=1+240 q+2160 q^{2}+\cdots$ and $E_{6}(z)=1-504 q+-16632 q^{2}+\cdots$.

We are ready to state our main theorem.

TheOREM 1.1. Let $k>4 r-4$ be an even positive integer such that $k \equiv 0 \quad(\bmod 4)$. For any $f=\sum_{n \geq 0} a_{f}(n) q^{n} \in M_{k}\left(\Gamma_{0}^{+}(5)\right) \cap \mathbb{Z}[[q]]$, we have that for each positive integer $\bar{b}$,

$$
a_{f E_{6}}\left(5^{b}\right) \equiv-a_{f}(0) \quad(\bmod 5) .
$$

\section{Proof of Theorem 1.1}

For each positive even integer $k>2$, let

$$
\begin{gathered}
E_{k}^{+}(z)=E_{k}+5^{k / 2} E_{k}(5 z), \\
E_{2}(z)=1-24 \sum_{n>0} \sigma_{1}(n) q^{n}, \quad E_{2}^{+}(z)=E_{2}-5 E_{2}(5 z),
\end{gathered}
$$

then $E_{k}^{+}(z)$ is a modular form for $\Gamma_{0}^{+}(5)$ of weight $k$ and $E_{2}^{+}(z)$ is a modular form for $\Gamma_{0}(5)$ (see [5, page 88]) whose the sign of the Fricke involution is -1 . Consequently $\left(E_{2}^{+}(z)\right)^{2}$ is a modular form for $\Gamma_{0}^{+}(5)$ of weight 4

Specially we have the following Fourier expansions:

$$
E_{4}^{+}(z)=26+240 q+\cdots, \quad\left(E_{2}^{+}(z)\right)^{2}=16+192 q+\cdots .
$$

Thus

$$
\Delta_{5}^{+}(z):=\frac{13\left(E_{2}^{+}(z)\right)^{2}-8 E_{4}^{+}(z)}{1576}=q+\cdots
$$

is a normalized cusp form for $\Gamma_{0}^{+}(5)$ of weight 4 . The below proposition guarantees that $\Delta_{5}^{+}(z)$ has no zero on $\mathbb{H}$.

Proposition 2.1. Let $f$ be a modular form for $\Gamma_{0}^{+}(5)$ of weight $k$, which is not identically zero. We have

$$
\sum_{p \in \Gamma_{0}^{+}(5) \backslash \mathbb{H}} e_{p} v_{p}(f)+v_{\infty}(f)=\frac{k}{4},
$$

where $1 / e_{p}$ is the cardinality of $\Gamma_{0}^{+}(5)_{p}$ and $v_{p}(f)$ is the order of a modular form $f$ at a point $p$.

Proof. See [4, Proposition 2.1]. 
We define a Hauptmodul $j_{5}^{+}(z)$ for $\Gamma_{0}^{+}(5)$ which plays an important role in this paper as follows

$$
j_{5}^{+}(z):=\frac{E_{4}^{+}(z)}{\Delta_{5}^{+}(z)}=\frac{1}{q}+\cdots .
$$

For any $f \in M_{k}\left(\Gamma_{0}^{+}(5)\right)$, we define

$$
W(f)=\frac{f}{\left(\Delta_{5}^{+}\right)^{r-1}} .
$$

To prove Theorem 1.1 we need the following proposition.

Proposition 2.2. $W$ is a vector space isomorphism from $M_{k}\left(\Gamma_{0}^{+}(5)\right)$ onto the space $R$ of polynomials in $j_{5}^{+}$of degree less than $r$.

Proof. For $d=0,1, \ldots, r-1$ the functions $\left(j_{5}^{+}\right)^{d}\left(\Delta_{5}^{+}\right)^{r-1} \in M_{k}\left(\Gamma_{0}^{+}(5)\right)$. Since $W\left(\left(j_{5}^{+}\right)^{d}\left(\Delta_{5}^{+}\right)^{r-1}\right)=\left(j_{5}^{+}\right)^{d}, W$ carries the subspace $Q$ of $M_{k}\left(\Gamma_{0}^{+}(5)\right)$ generated by the modular forms $\left(j_{5}^{+}\right)^{d}\left(\Delta_{5}^{+}\right)^{r-1}$ isomorphically onto $R$. Hence $\operatorname{dim} Q=r$ which implies that $Q=M_{k}\left(\Gamma_{0}^{+}(5)\right)$.

We are ready to prove Theorem 1.1. We note that two functions

$$
\frac{-1}{2 \pi i} \frac{d j_{5}^{+}(z)}{d z}=\frac{26}{q}+\ldots
$$

and

$$
\frac{E_{6}^{+}(z)}{\Delta_{5}^{+}(z)}=\frac{126}{q}+\cdots
$$

are weakly holomorphic modular forms for $\Gamma_{0}^{+}(5)$ of weight 2 . We note that $M_{2}\left(\Gamma_{0}^{+}(5)\right)=\{0\}$. These imply that

$$
\frac{-63}{26 \pi i} \frac{d j_{5}^{+}(z)}{d z}=\frac{E_{6}^{+}(z)}{\Delta_{5}^{+}(z)} .
$$

Moreover, we have that

$$
j^{m} \frac{d j_{5}^{+}(z)}{d z}=\frac{1}{m+1} \frac{d\left(j_{5}^{+}(z)\right)^{m+1}}{d z} \quad(m \in \mathbb{Z}, m \geq 0) .
$$

Since the constant term in the Fourier expansion of $\frac{d\left(j_{5}^{+}(z)\right)^{m+1}}{d z}$ is zero, by linearity it follows that

$$
\left(j_{5}^{+}\right)^{5^{b}-r} \frac{-63 f}{26 \pi i\left(\Delta_{5}^{+}\right)^{r-1}} \frac{d j_{5}^{+}}{d z}
$$


has constant term zero. Thus we have that the constant term of

$$
\begin{aligned}
\left(j_{5}^{+}\right)^{5^{b}-r} \frac{-63 f}{26 \pi i\left(\Delta_{5}^{+}\right)^{r-1}} \frac{d j_{5}^{+}}{d z} & \equiv \frac{f E_{6}}{\Delta_{5}^{+}\left(5^{b} z\right)} \\
& \equiv\left(\sum_{n \geq 0} a_{f E_{6}}(n)\right)\left(q^{-5^{b}}+1+\ldots\right) \\
& \equiv \cdots+\left(a_{f E_{6}}\left(5^{b}\right)+a_{f E_{6}}(0)\right)+\cdots(\bmod 5)
\end{aligned}
$$

is zero modulo 5 which means

$$
a_{f E_{6}}\left(5^{b}\right) \equiv-a_{f E_{6}}(0) \equiv-a_{f}(0) \quad(\bmod 5) .
$$

\section{References}

[1] Y. J. Choie, W. Kohnen, and K. Ono, Linear relations between modular form coefficients and non-ordinary primes, Bull. London Math. Soc. 37 (2005), no. 3, 335-341.

[2] T. Miyake, Modular forms, Translated from the 1976 Japanese original by Yoshitaka Maeda. Reprint of the first 1989 English edition. Springer Monographs in Mathematics. Springer-Verlag, Berlin, 2006. $\mathrm{x}+335$ pp. ISBN: 9783-540-29592-1; 3-540-29592-5.

[3] K. Ono, The web of modularity: arithmetic of the coefficients of modular forms and q-series, CBMS Regional Conference Series in Mathematics, 102. Published for the Conference Board of the Mathematical Sciences, Washington, DC; by the American Mathematical Society, Providence, RI, 2004. viii+216 pp. ISBN: 0-8218-3368-5.

[4] J. Shigezumi, On the zeros of the Eisenstein series for $\Gamma_{0}^{*}(5)$ and $\Gamma_{0}^{*}(7)$. Kyushu J. Math. 61 (2007), no. 2, 527-549.

[5] W. Stein, Modular forms, a computational approach, With an appendix by Paul E. Gunnells. Graduate Studies in Mathematics, 79. American Mathematical Society, Providence, RI, 2007. xvi+268 pp. ISBN: 978-0-8218-3960-7; 0-82183960-8.

Department of Mathematics Education

Dongguk University-Gyeongju

Gyeongju 780-714, Republic of Korea

E-mail: young@dongguk.ac.kr 